Historic, Archive Document

Do not assume content reflects current scientific knowledge, policies, or practices. 



\section{SPECIAL SALE LIST}

19290

\section{Trees - Shrubs - Flowers Bulbs - Plants}

\section{The \\ E. F. Weaver Nurseries and Greenhouse}

37 Years in Wichita

GROWERS AND BUILDERS OF BEAUTY

1900 Cast Central Ave. Phone Market 1742

\section{Wichita, Kansas}

Visit or phone our Nurseries for suggestions and planting advice. Let us help you, our experience will safeguard you against mistakes, dissappointments and unnecessary expense. Ask us for advice, there is no cost or obligation. If desired our experienced landscape men can do your planting. Estimates free.

\section{EVERGREENS-Everlasting Beauty, Rich in Color.}

The E. F. Weaver Nurseries field count of growing Evergreens, all kinds, July 1928, showed 21,450 trees. Arrange at once to visit our display and sales grounds at 1900 East Central. You will admire the impressive Evergreen showing. See with your own eyes the perfect specimens of Bakers, Compactas, Pyramidals, Globes, Bonitas, Rosedales, Orientals, Pfitzers, Platt River Cedar, Red Cedar, Prostrate and Spiney Greek Junipers, Mugho, Srotch and Dwarf Pines. In fact we pride ourselves for having everything there is for high class landscape work. You will be surprised at the moderate prices.

\section{ORNAMENTAL \& FLOWERING SHRUBS.}

Include all proven and hardy kinds for Southwest conditions. Althea, Almond, Buddles or Butterfly Bush, Japanese Barberry, Dogwoods red and yellow, Japanese Quince, Desmodium or Bush Clover, Deutzia, Russian Olive, Euonymus, Forsythia or Golden Bell, Hibiscus, Rose Mallows or Giant Mallow Marvels, Hydrangeas, Bush Honeysuckles, Ligustrum Ibota, Lilacs, Mock Orange, Syringas, Pussy Willows, Cutleaf Rhus or Sumac, Golden, Sweet American ard Fern Leaf Elder, Caragana or Siberian Pea, Snowbali, High Bush Cranberry, Red and White Snowberry, Spiraea Van Houteii, Spiraea Thumbergii, Red and Pink Dwarf Spiraeds, Tamarix, Vitex, Weigelia, etc. Price on all shrubs named above:

Strong Heavy, 2-yr., 2 to 4 feet tall_._.....each 50c; any asst. 5, $\$ 2.00$

Extra Heavy, 3-yr., landscape specimens, 3 to 5 feet_each $75 \mathrm{c} ; \quad 5 \$ 3.00$

New Red Leaf Barberry and Callacarpa Purperea_._._._._._each 90c

SPECIAL NOTICE--Quantity Prices permit you to make any assortment desired, say one each of several varieties and club together at the quantity prices. 
Boston or Japan Ivy, Englemans Ivy, Bignonia Grandiflora, Bitter Sweet, White Star Clematis, Halls Japan Honeysuckle, Matrimony Vine, Wisteria. Strong, 2 year, each 50c; 5, \$2.00; Heavy, 3 year each $75 c ; 5 \$ 3.00$.

Large Flowering Clematis, Purple Violet, Red or White, and Lonercera Heckrotte, each $75 c$.

Hardy Blue Myrtle or Vinca, English or Evergreen Ivy, American Ivy or Virginia Creeper, each $25 c ; 5, \$ 1.00$.

Hardy Live-over-winter Sweet Peas, Red, Pink, White Mix, Chinese Cinnamon Vines, Blackeyed Susan, Climbing Moonvines, Variagated Vinca, Asparagus, Sprengerii or Fern, Wandering Jew. Each 15c; 8, $\$ 1.00$.

Datura or Bush Moonvine, Maderia or Sweet Mognonette Vine, each $10 \mathrm{c} ; 12, \$ 1.00$.

HARDY LIVE OVER WINTER PERENNIAL FLOWERS-All 2 yr.

Hardy Blue Ageratum, Anchusa, Artemisia Silver King, Asclepias or Butterfly Flower, Hardy Asters, Boltonia, Baby's Breath, Blazing Star, Bluebells, Campanulas or Canterbury-bells, Chrysanthemums, in button Pompom and large flowering types, colors Red, Yellow, White, Pink, Bronze and various shades, Catnip, Cat-tails, Hardy Carnations, Grass Pinks, Columbines, Centaureas or Hardy Cornfiower, Correopsis, Chinese Lantern Plants, Shasta Daisy, Memorial Daisy, Painted Daisy, Hardy Larkspur or Delphinum, Dianthus or Sweet William, Eshinops, Eryngium, Giant Summer Hyacinths, Gaillardia, Golden Glow, October Golden Glow, Oriental Poppys, Hardy Ornamental Grasses, Pampass Grass, Maiden Grass, Stripped Japanese, Zebra Grass, Ribbon Grass, Helianthus, Hibiscus, Hollyhocks, Iris in variety, Lythrus or Everlasting Pcas, Madonna Lily (in pots) Double and Single Tiger Lily Regale or Royal Lily, Milk and Wine Lily, Spider Lily, Lily of Valley, Red Canada Lily, Turks Cap Lily, Day Lillies, Liatris or Gay Feather, Monarda, Phlox all Color's, Physostegia, Pyrethrum, Hardy Sweet Peas, Sopanaria, Secum, Tritoma or Red Hot Poker, Vinca or Hardy Myrtle, Yucca. Prices on all hardy perennials named above. Each 25c; any assortment, 5, $\$ 1.00$.

Peonies. Red, each 75c; Pink and White, each 50c; 5, \$2.00.

Bleeding Heart, Giant Reed or Bambo Grass, Hardy Red Salvia. Each $50 \mathrm{c}$; any assortment, $5, \$ 2.00$

\section{SHADE AND ORNAMENTAL TREES.}

American Elm. $10-12$ ft. $\$ 1.50 ; 12-15$ ft. $\$ 2.50 ; 3-4$. in Caliper, $\$ 3.50$.

Alianthus or Prairie Palm. 2-3 ft. $25 \mathrm{c} ; 4-5 \mathrm{ft} .50 \mathrm{c} ; 6-8 \mathrm{ft}$. $75 \mathrm{c}$.

Silver or Soft Maple Shade Trees. $8-10 \mathrm{ft} . \$ 1.00 ; 10-12 \mathrm{ft}$. $\$ 1.50 ; 1 \frac{1}{2}-2$

in. Caliper $\$ 2.50 ; 2-2 \frac{1}{2}$ in. $\$ 3.50$.

European White Birch. 6-8 ft. $\$ 2.00$

Catalpa Speciosa. $2-2 \frac{1}{2}$ in. caliper $\$ 2.50 ; 21 / 2-3$ in. $\$ 3.50$.

Umbrella Catalpa. $3-4 \mathrm{ft}$. 'stems $\$ 1.75 ; 4-5 \mathrm{ft}$. stems $\$ 2.25 ; 5-6 \mathrm{ft}$. stems $\$ 2.50 ; 6-7 \mathrm{ft}$. stems $\$ 3$.

China-berry or Texas Umbrella Tree. 3-4 ft., each 75c.

CHINESE ELM-The only shade tree to plant, rapid growth, very hardy, will make a fine shade tree in three years. Ask for special circular and prices.

Lombardy Poplars. $5-6$ ft. $50 \mathrm{c} ; 6-8 \mathrm{ft} .75 \mathrm{r} ; 8-10 \mathrm{ft} . \$ 1 ; 10-12 \mathrm{ft} . \$ 1.50$; $12-15 \mathrm{ft} \$ 2.50$.

New Chinese Lombardy. Special sale. $5-6 \mathrm{ft} .50 \mathrm{c} ; 6-8 \mathrm{ft} .75 \mathrm{c}$.

Carolina Poplar. 4-5 ft. $35 \mathrm{c} ; 5-6 \mathrm{ft} .50 \mathrm{c}$.

Sweet Gum, red foliage B \& B. $10-12 \mathrm{ft} . \$ 3.50$.

Red Bud. Specimens B \& B. $10-12 \mathrm{ft}$. $\$ 3.50$.

Teas Weeping Mulberry, Cut Leaf Weeping Birch. 5-6 ft., 4-yr. heads. Each $\$ 4.50$.

Weeping Willow Wisconsin type. 10-12 ft. $\$ 2.50$

Niobe Weeping Willow. Fine specimens B\&B. 10-12 ft. $\$ 3.50$.

Russian Olive. Valuable and decorative. $3-4 \mathrm{ft} .75 \mathrm{c} ; 4-5 \mathrm{ft}$. $\$ 1.25 ; 6-8$ tit. $\$ 2.50$

\section{NUT BEARING TREES-(All Budded and Grafted Stock)}

Pecans, Soft Shell, Stewart and Schley, 3-4 ft. $\$ 1.05$.

Paper Shell Pecans. Extra fine. Best named varieties. 6-8 ft. Each $\$ 3$. American Sweet Chestnuts. 5-6 ft, each $\$ 1.50 ; 6-8 \mathrm{ft}$. each $\$ 2.00$ 


\section{SUMMER FLOWERING BULES}

Cinnamon Vine Bulbs. 3, 25; 15, $\$ 1$.

Caladium or Elephant Ears. Large size. Each 35c; extra size 50c.

Giant Summer Hyachinthas. $12,35 \mathrm{c} ; 50, \$ 1 ; 100, \$ 1.75$.

Lilies, all varieties, see Hardy Perennials.

Maderia Vine Bulbs, extra large. 3, 25c; 15, $\$ 1$.

Tube Roses, Mexican Everblooming, very fragant. 12, 50c; 30, $\$ 1.00$.

Cannas, large flowering, dwarf assorted, all colors. 6, 25c; 25, \$1.00.

President, Rich Red, the best. $6,25 c ; 25, \$ 1.00$.

Austria, Yellow, Red Flecked. 6, 25c; 25, \$1.00.

Red King Humbert, bronze foliage. $4,25 \mathrm{c} ; 20, \$ 1.00$.

Hungaria, Best Pink. 2, 25c; $8, \$ 1.00$.

Snow Queen, Pure White. 2, 25c; 8, $\$ 1.00$.

Wyoming, Rich Orange. $4,25 \mathrm{c} ; 20, \$ 1.00$.

Dahlias. Extra fine cut flower mixture. 2, 25c; 10, $\$ 1.00$.

Gladioli. King of flowers, 20 named varieties, all flashy colors in a wonderful assortment. $6,25 \mathrm{c} ; 30, \$ 1.00$.

Kansas Gay Feather Bulbs. Fach 25c; 5, $\$ 1.00$.

\section{HEDGING PLANTS-}

Hardy Amoor River Privett North, and Hardy Ibolium Privett. 12-15 inches, per $100, \$ 4 ; 15-18$ inches, per $100, \$ 6 ; 18-24$ in., per $100, \$ 8 ; 3-4$ feet, per 100, $\$ 15$.

(We do not handle California Privett as it is not hardy here.) $100, \$ 4.00$.

HEDGE MULBERRY-12-18 inches, per 100, \$3; 18-24 inches, per

EVERGREENS for hedging. Chinese Arborvitea. 12-18 in., each 25c; $100, \$ 20.00 ; 4-5$ feet, each $80 \mathrm{c} ; 100, \$ 75$.

\section{GARDEN PLANTS-}

Asparaguis Roots, Mary Washington or Palmetto, extra heavy 2 yr., 25, 50 ; ; by mail, prepaid, $25, \$ 1.00$.

New Giant Victoria Rhubarb, stands our hot dry summers, 1 yr., undivided crowns, $6,25 \mathrm{c} ; 15,50 \mathrm{c} ; 30, \$ 1$. $3 \mathrm{yr}$. divisions, $3,25 \mathrm{c} ; 6,50 \mathrm{c} ; 12, \$ 1.00$. Delivered anywhere prepaid.

Mammoth French White Jerusalem Artichokes. Peck, $\$ 1.00, \mathrm{Bu} ., \$ 3.00$

Horseradish roots, Imp. Maliner Kren, Root Sets. 12, 25c; 100, $\$ 1.75$; Crown Sets $10,25 c ; 100, \$ 2.00$.

Holts Mammoth Garden Sage. Each 25c.

Strawberry Plants, tried and proven kinds.

Senator Dunlap or Aroma 150, $\$ 1$.

Progressive Everbearing. 75, $\$ 1$. Mastodon Everbearing 50, $\$ 1.00$.

Strawberries delivered anywhere prepaid.

VEGETABLE PLANTS-All Sash Grown with heavy roots, well hardened.

Special Plant Collection-50 Cabbage, 50 Tomato, 10 Sweet Peppers, 5 Egg Plant. All $\$ 1.00$. Prepaid anywhere.

Cabbage Plants-Early Jersey Wakefield, Charleston, all seasons. Sure head. Early Flat Dutch and Copenhagen Market. 12 dozen growing plants in flats, 90c. Pulled, dozen, $15 \mathrm{c} ; 25,25 \mathrm{c} ; 100,60 \mathrm{c} ; 1000, \$ 4.50$.

Tomato Plants-Earliana, Chalks Jewell, Burbank, John Bear, Early Detroit, Bonny Best, Red Head, Red Rock, New Stone, Dwarf Stone, Baltimore, Marglobe, Tree, Acme, Beefsteak, Matchless, Kansas Standard, Pondorosa, Golden Queen, Yellow Pear, Yellow Plum. 12 dozen growing plants in flats 90c. Pulled, dozen, $15 c ; 25,25 c ; 100,60 c ; 1000, \$ 4.50$.

Celery Plants, White Plume and Golden. Dozen 25c.

Cauliflower Plants, Early Snowball. Dozen 20c.

Pepper Plants-Goliath, Giant Crimson, Chinese Giant, Bull Nose, Royal King, Ruby King, Sweet Pimento, Anabeim Chili and Red Hots. 12 dozen growing piants in fiats, $\$ 1$. Pulled plants, dozen, $20 \mathrm{c} ; 25,35 \mathrm{c} ; 100, \$ 1.00$; $1000, \$ 8.00$.

Egg Plants-Black Beauty and Imp. New York Purple. 12 dozen growing plants in flats, $\$ 1$. Pulled plants, dozen, 20c; $25,35 \mathrm{c} ; 100, \$ 1 ; 1000, \$ 8$.

Sweet Potato Plants-Yellow Jersey, Nancy Hall, Porto Ricans, Triumphs, 20, 25c; 50, 50c; $100, \$ 1 ; 300, \$ 2 ; 1000, \$ 6$. 


\section{BEDDING PLANTS.}

Our new sash greenhouses produce high grade plants for Urns, Window and Porch Boxes, and bedding out. Such as Sweet Alyssum, Ageratum, Daisies of all kinds, Snapdragons, Verbenas, Petunias in all fine varieties, Asters, Calendulas, Cornflowers, Annual Mums, Celosia or Cockscomb, Summer Cypress, Marigold, Larkspur, Mourning Bride. Nicotina, Drummodi Phlox, Double Moss or: Portulaca, Red Salvia, Giant Zinnia, Strawflowers or Everlastings, Bachelor Buttons, Flowering Vinca or Perewinkle, Immortelles. Each kind, Doz. 25c, Any assortment of not less than 5 of a kind, 100, $\$ 2.00$.

\section{POTTED PLANTS.}

Dusty Miller, Santolinas, Alternantheras, Coleus, White Artemsia, Vinca, Wandering Jew, Begonias, Cinerarias, Double and Ruffled Monster Petunias, Beauty of Oxford Verbenas, and many other varieties at popular prices.

OLEANDERS-New flashy colors, bushy 3 to $4 \mathrm{ft}$. blooming plants, $\mathrm{B} \& \mathrm{~B}$, each $\$ 2.50$. In tubs, $\$ 3.50$.

URNS, PORCH AND WINDOW BOXES-in variety at bargain prices. Come and see them.

PLANTING SERVICE-For Urns, Porch and Window Boxes and Beddịng Plants at cost.

It is a pleasure to assist you in planning how to beautify your home, there is no charge or obligation for this advice. Landscape estimates free.

\section{FRUIT TREES, BERRIES \& GRAPES-}

APPLE TREES-Ben Davis, Jonathans, Deiicious, Wealthy, Early Harvest, Winesap, Staymans, Gano, Grimes Golden, Yellow Transparent, Hyslop Crab, 5-6 ft. trees, each 75c; 5, \$3.50.

CHERRY TREES-Dyehouse, Early Richmond, Montmorency, large 5-6 ft. trees, each $75 c ; 5, \$ 3.50$.

PLUM TREES-Burbank, Blue Damson, Lombard, Abundance, Large 5-6 fit. trees, each $65 \mathrm{c} ; 5, \$ 3.00$.

PEACHES-Elberta, Carmen, Champion, Chinese, Hale, Red Bird, 4-5 ft. trees, each $50 \mathrm{c} ; 5, \$ 2.00$.

CONCORD GRAPES-The stock we offer is the finest in the U. S. Heavy, 3-yr., fruiting size, $2,25 \mathrm{c}$ : $10, \$ 1.00 ; 100, \$ 7 ; 1000, \$ 50$.

CURRANTS-Wilder Red. Each, 25c; 5, $\$ 1.00$.

RASPBERRIES-Cumberland, Kansas, Latham, St. Regis, 3, 25c; $12, \$ 1$. BLACKBERRIES--Early Harvest, Snyder, Eldorado. 3, 25c; 12, $\$ 1.00$. GOOSEBERRIES-Carrie, Pearl, Houghton. Each 25॰; 5, \$1.00.

\section{ROSES}

No one has any better Roses for Kansas conditions. Ask any of our customers who have tried them. All strong 2-yr. Field Grown Blooming Size Bushes:

Los Angeles, White Killarney, J. L. Mock, Mrs. Aaron Ward, Frau Karl Druschki, Columbia, Gruss An Teplitz, Souv. De Claud, Pernet, Etoile De France, Pink Radiance, Sunburst, Mme. Butterffy, Red Radiance, Shell Radiance, K. A. Victoria, La France, Bessie Brown, Carolina Testout, Francis Scott Key, Lady Hillingdon. Luxenburg. Cl. American Beauty, Paul Neyron, Gen. Jack, Rugosa, Rosa Blanda.

Your selection of any variety or color, each labeled, 50c each. Any assortment, $5, \$ 2$.

EXTRA SPECIAL-Our selection of 5 separate varieties from above list, all 2-yr. field grown, to include White, Pink, Red, and Yellow, all tied in one bundle, not labeled, $\$ 1$. Only a few thousand to go at these prices, better send in your order. 